\title{
ENSAYO BIOGEOGRAFICO DE LA PTERIDOFLORA VALENCIANA
}

\author{
Juan J. HERRERO-BORGOÑON, Ana Mª IBARS, \\ Julio IRANZO, Mercedes VILAR, Alfonso PINA, \\ Juan C. GARCIA-VERDUGO \& A. Enrique SALVO
}

RESUMEN: En el presente trabajo se realiza un análisis comparativo de las pteridofloras de los diiferentes sectores fitogeográficos (sensu Costa, 1986) que integran la Comunidad Valenciana.

Palabras clave: Pteridófitos, biogeografía, Comunidad Valenciana.

ABSTRACT: The present paper deals with the biogeography and of pteridophytes in the Comunidad Valenciana (Levante, Spain), following the present chorological division of this area.

Key words: Pteridophytes, biogeography, Comunidad Valenciana.

\section{INTR ODUCCION}

Se pretende con este trabajo realizar una aproximación biogeográfica a la pteridoflora valenciana, con especial hincapié en la variación de los índices aplicables usualmente en este tipo de estudios en relación con la ordenación latitudinal de las unidades corológicas. Para ello se ha seguido fundamentalmente la sectorización corológica más reciente de dicha Comunidad (Costa 1986). En el presente trabajo únicamente nos referiremos a cuatro de los sectores (Setabense, Valenciano-Tarraconense, Alicantino y Maestracense) por cuanto el quinto (Sector Manchego) queda, por su situación, fuera de nuestro objetivo de análisis latitudinal.

\section{A TALOGO}

En la Tabla I se expone el catálogo de los táxones cuya cita ha sido constatada hasta el momento en la Comunidad Valenciana. El significado de las columnas en dicha Tabla es el siguiente: 
Tabla 1.

\begin{tabular}{|l|}
\hline \\
\hline Adiantum capillus-veneris \\
\hline Anogramma leptophylla \\
\hline Asplenium adiantum-nigrum \\
\hline Asplenium ceterach ceterach \\
\hline Asplenium fontanum fontanum \\
\hline Asplenium foreziense \\
\hline Asplenium onopteris \\
\hline Asplenium petrarchae petrarchae \\
\hline Asplenium ruta-muraria ruta-muraria \\
\hline Asplenium sagittatum \\
\hline Asplenium scolopendrium \\
\hline Asplenium septentrionale septentrionale \\
\hline Asplenium trichomanes pachyrachis \\
\hline Asplenium trichomanes quadrivalens \\
\hline Athyrium filix-femina \\
\hline Botrychium lunaria \\
\hline Cheilanthes acrosticha \\
\hline Cheilanthes maderensis \\
\hline Cheilanthes tinaei \\
\hline Cosentinia vellea bivalens \\
\hline Cosentinia vellea vellea \\
\hline Cystopteris fragilis \\
\hline Dryopteris filix-mas \\
\hline Equisetum arvense \\
\hline Equisetium palustre \\
\hline Equisetum telmateia \\
\hline Hippochaete ramosissima \\
\hline Isoetes velatum \\
\hline Marsilea batardae \\
\hline Notholaena marantae \\
\hline Ophioglossum vulgatum \\
\hline Polypodium cambricum serratum \\
\hline Polypodium vulgare \\
\hline Polystichum aculeatum \\
\hline Polystichum setiferum \\
\hline Pteridium aquilinum aquilinum \\
\hline Pteris vittata \\
\hline Selaginella denticulata \\
\hline Thelypteris palustris \\
\hline TOTAL \\
\hline
\end{tabular}
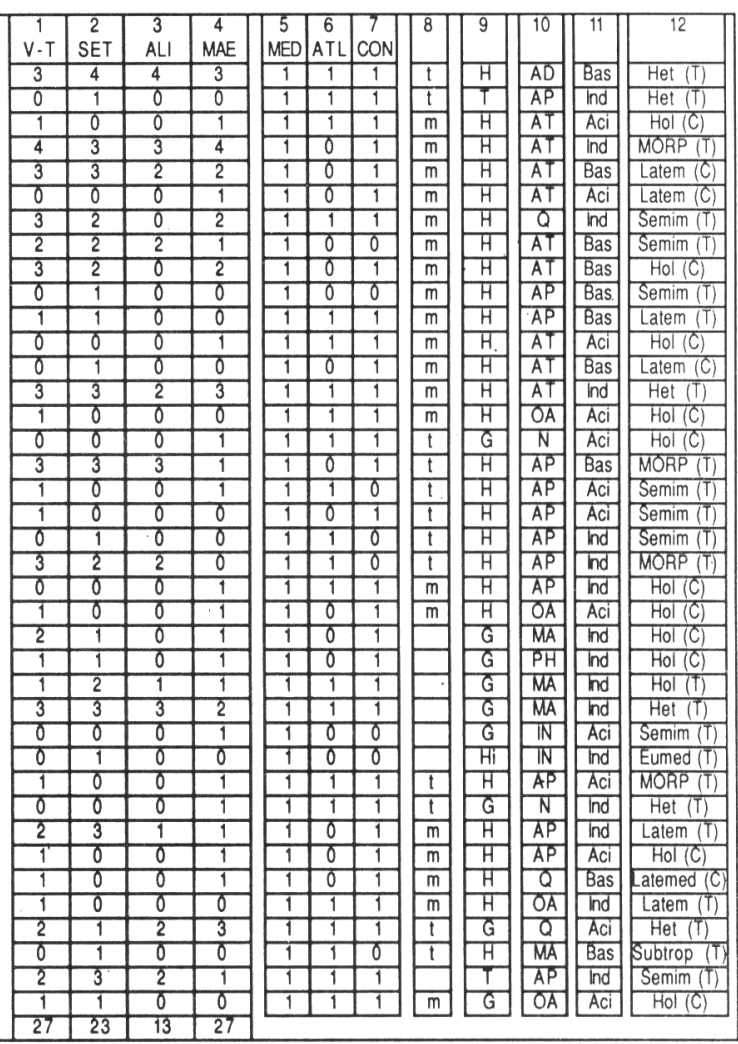

1-4: Frecuencia de aparición por sectores corológicos. Se consideran seis rangos posibles, indicados por $0=$ ausente, $1=$ muy raro, $2=$ raro, $3=$ ocasional, $4=$ frecuente, $5=$ abundante.

5-7: Carácter biogeográfico. Expresa la presencia (1) o ausencia (0) de los táxones considerados en las regiones Mediterránea (MED), Macaronésica (ATL) y Eurosiberiana (CON).

8: Carácter monoleta-trileta. Expresa el tipo de laesura que presentan las especies de filicofitinas: trilaesuradas $(\mathrm{t})$ y monolaesuradas $(\mathrm{m})$.

9: Biótipo. Se sigue la clasificación de Raunkier, estimándose en cuatro los biotipos presentes: Geófitos $(\mathrm{G})$, Hemicriptófitos $(\mathrm{H})$, Terófitos $(\mathrm{T})$ e Hidrófitos (Hi).

10: Ecosistemas preferenciales. Se consideran 9 tipos de ecosistemas, consignándose aquel en que aparece más frecuentemente representado el taxon referido. Las abreviaturas corresponden a: $(A D)$ grietas y fisuras de rocas calizas rezumantes; (AP) grietas terrosas húmedas y umbrías; (AT) fisuras de rocas verticales; (IN) praderas de nanojuncos temporal o permanentemente sumergidas; (MA) pastizales de suelos húmedos; $(\mathrm{N})$ pastizales de alta montaña; $(\mathrm{OA})$ bosques ribereños; $(\mathrm{PH})$ comunidades de 
bordes de zonas húmedas con carrizos; (Q) bosques.

11: Comportamiento edáfico. Se distribuyen los táxones en tres clases: Acidófilos (Aci), Basófilos (Bas) e Indiferentes (Ind), si bien hay que tener en cuenta que dicho comportamiento edáfico puede verse afectado por otros factores como la distribución altitudinal.

12: Tipos biogeográficos. Siguiendo a Pichi-Sermolli (1979) y a PichiSermolli, España \& Salvo (1987) se establecen las siguientes categorías: un primer grupo cuyos táxones ya estaban presentes en la Región Mediterránea durante el Terciario, y que se subdividiría, a su vez, en: (A) Heterocóricos, (B) Circumboreales, (C) Mediterráneos y de regiones orientales próximas, (D) Latemediterráneos, (E) Subtropicales, (F) Semimediterráneos y (G) Eumediterráneos. Por otro lado se consideran aquellos táxones presentes en la Región desde el Cuaternario, subdivididos en: $(\mathrm{H})$ Circumboreales, (I) Latemediterráneos.

Para el estudio del espectro fitogeográfico, estos grupos se reúnen de la siguiente manera: Heterocóricos, A; circumboreales, $\mathrm{B}+\mathrm{H}$; Submediterráneos, $\mathrm{C}+\mathrm{D}+\mathrm{F}+\mathrm{I}$; Subtropicales, E; y Eumediterráneos, G.

\section{ANALISIS PTERIDOGEOGRAFICO}

\section{CARACTER PTERIDOFLORISTICO.}

A. Indice de similitud con las regiones próximas (ISRP).

Siguiendo a Salvo (1982) y Salvo \& Cabezudo (1984) se han calculado los índices de similitud de la pteridoflora de cada uno de los sectores comentados con cada una de las regiones biogeográficas en que se encuentran los táxones utilizados en este estudio. Los resultados están expresados en la Tabla II.

De ellos se deduce el carácter marcadamente Mediterráneo de la pteridoflora valenciana, si bien es interesante señalar algunos matices distintivos, como las diferencias entre las tendencias secundarias que son atlánticos en los sectores Setabense y Alicantino, y continental en los sectores Valenciano-Tarraconense y Maestracense. Estas tendencias pueden ser achacadas a las diferencias climáticas provocadas por la mayor altitud media de los dos últimos sectores.

Tabla II: ISRP de los distintos sectores y regiones

\begin{tabular}{lcccc}
\hline \hline & VAL-T & SET & ALICANT & MAEST \\
\hline R. MEDITERRANEA & 0.44 & 0.40 & 0.22 & 0.44 \\
R. MACARONESICA & 0.27 & 0.24 & 0.15 & 0.26 \\
R. EUROSIBERIANA & 0.29 & 0.22 & 0.13 & 0.29 \\
\hline
\end{tabular}

B. Indice de similitud entre sectores.

También se han calculado los índices de similitud de los distintos sectores entre sí (Tabla III), obteniéndose resultados que concuerdan con lo expuesto anteriormente, ya que las menores similitudes aparecen en los sectores Alicantino y Seta- 
bense con respecto al Maestracense, mientras que éste aparece íntimamente ligado al Valenciano-Tarraconense, al igual que el Alicantino y el Setabense entre sí. Aparece también una fuerte relación entre los sectores Valenciano-Tarraconense y Setabense, lo que no es de extrañar, dada su contigüidad geográfica.

Tabla III: ISRP de los distintos sectores entre sí.

\begin{tabular}{lccc}
\hline \hline & SET & ALICANT & MAEST \\
\hline VAL-T. & 0.70 & 0.61 & 0.77 \\
SET & $\cdot$ & 0.66 & 0.58 \\
ALIC & $\cdot$ & $\cdot$ & 0.56 \\
\hline
\end{tabular}

C. Indice $\mathrm{m} / \mathrm{t}$.

Este índice, propuesto por Ito (1972) y desarrollado por Pichi-Sermolli, España \& Salvo (1988), está directamente relacionado con la latitud, la altitud y Ia pluviosidad de la zona considerada. El valor del índice $\mathrm{m} / \mathrm{t}$ calculado para los distintos sectores se puede observar en la Tabla IV.

Tabla IV: Indice $\mathrm{m} / \mathrm{t}$ de los distintos sectores.

\begin{tabular}{lrrl}
\hline \hline & $\mathrm{m}$ & $\mathrm{t}$ & $\mathrm{m} / \mathrm{t}$ \\
\hline VALENCIANO-T & 15 & 7 & 2,14 \\
SETABENSE & 11 & 7 & 1,57 \\
ALICANTINO & 5 & 4 & 1,25 \\
MAESTRACENSE & 14 & 7 & 2,00 \\
\hline
\end{tabular}

Así tenemos que el sector Maestracense y Valenciano-Tarraconense, con una mayor latitud que los otros, poseen índices más elevados. Sin embargo, aparece aquí una anomalía, ya que el sector Maestracense, de mayor altitud media y pluviosidad que el Valenciano-Tarraconense, debería poseer un índice más elevado, cosa que no ocurre. Dicha anomalía podría deberse a los problemas intrínsecos que presenta la aplicación del índice m/t a pteridofloras pobres (Salvo, García-Verdugo \& Pina, en prensa), que provocan, a veces, la aparición de resultados impredecibles y poco discriminatorios. Por otra parte, el resto de los resultados es perfectamente coherente, ya que el menor índice lo presenta el sector Alicantino, que es el de menor latitud, altitud y pluviosidad, siendo el del sector Setabense intermedio, tal como puede esperarse de sus características.

\section{ANALISIS ECOLOGICO.}

\section{A. Espectro biologico.}

Como puede deducirse de la Tabla V, la forma biológica más frecuente es en todos los casas la hemicriptofítica, siguiéndole la geofítica y la terofítica, ésta última con muy escasa representación. Finalmente es en el sector Setabense en el único en 
el que se encuentra representación hidrofítica.

Tabla V: Espectro biológico.

\begin{tabular}{lcccc}
\hline \hline & HEMICRIP & GEOFITO & TEROFITO & HIDROFITO \\
\hline VALENCIANO-T & 74,1 & 22,2 & 3,70 & 0,00 \\
SETABENSE & 62,5 & 25,0 & 8,33 & 4,17 \\
ALICANTINO & 66,7 & 25,0 & 8,33 & 0,00 \\
MAESTRACENSE & 66,7 & 29,6 & 3,70 & 0,00 \\
\hline
\end{tabular}

\section{B. Espectro fitocenológico.}

Los resultados para los 8 tipos de ecosistemas están reflejados en la Tabla VI.

De ellos se deduce que los más habituales son las grietas terrosas húmedas y umbrías y las fisuras de rocas verticales, que en todos los sectores alcanzan bastante más del $50 \%$ del total, llegando en algunos casos, como el Alicantino, hasta el $66 \%$. Tras estos, el ecosistema con más representatividad pteridofítica son los pastizales de suelos húmedos (que oscilan entre el 11.1 y el 16.7\%, según el sector), salvo en el sector Valenciano-Tarraconense, donde resultan ser los bosques ribereños. En general, los ecosistemas de bosque pueden considerarse también importantes, puesto que cuentan con porcentajes entre el 8.33 y el $11.1 \%$.

Tabla VI: Espectro fitocenológico.

\begin{tabular}{lrrrr}
\hline \hline & VAL-T & SET & ALIC & MAEST \\
\hline AD & 3.7 & 4.17 & 8.33 & 3.70 \\
AP & 33.3 & 33.30 & 33.30 & 25.90 \\
AT & 22.2 & 25.00 & 33.30 & 29.60 \\
IN & 0.0 & 4.17 & 0.00 & 3.70 \\
MA & 11.1 & 16.70 & 16.70 & 11.10 \\
N & 0.0 & 0.00 & 0.00 & 7.41 \\
OA & 14.8 & 4.17 & 0.00 & 3.70 \\
PH & 3.7 & 4.17 & 0.00 & 3.70 \\
Q & 11.1 & 8.33 & 8.33 & 11.10 \\
\hline
\end{tabular}

C. Espectro edáfico.

La gran mayoría de los táxones considerados en los cuatro sectores pueden ser calificados de indiferentes respecto al sustrato (Tabla VII), llegando la proporción al $58.3 \%$ en el sector Alicantino. Es de destacar la escasa representación de táxones acidófilos en los sectores Setabense y Alicantino (8.33\% en ambos casos) en comparación con los sectores Valenciano-Tarraconense y Maestracense (33.3 y 37\% respectivamente). Por contra, los táxones basófilos están peor representados en estos últimos sectores que en los primeros, si bien la diferencia no es tan evidente. Hay que tener en cuenta no obstante que, como ya se indicó anteriormente, estos resultados pueden verse afectados por factores ajenos al propio sustrato, como la distribución altitudinal, que, en algunos casos, pueden llegar a enmascarar de manera notable los resultados. 
Tabla VII: Espectro edáfico.

\begin{tabular}{lcccc}
\hline \hline & VAL-T & SET & ALIC & MAEST \\
\hline ACIDOFILOS & 33.3 & 8.33 & 8.33 & 37.0 \\
BASOFILOS & 25.9 & 37.50 & 33.30 & 37.0 \\
INDIFERENTES & 40.7 & 54.20 & 58.30 & 40.7 \\
\hline
\end{tabular}

\section{ANALISIS COROLOGICO.}

A. Espectro fitogeográfico.

De los cinco grupos considerados (Tabla VIII), el predominio absoluto para todos los sectores corresponde a los táxones Submediterráneos, si bien los componentes secundarios varían en general para cada sector, siendo los más importantes los táxones Circumboreales en los sectores Valenciano-Tarraconense y Maestracense, los Heterocóricos en el Alicantino, y los Heterocóricos y Circumboreales conjuntamente en el Setabense. Hay que hacer notar también que es en este último sector donde únicamente aparecen, aunque en escasa medida, táxones Eumediterráneos y Subtropicales.

Tabla VIII: Espectro fitogeográfico.

\begin{tabular}{lrrrr}
\hline \hline & VAL-T & SET & ALIC & MAEST \\
\hline HETEROCORICOS & 14.8 & 20.80 & 33.30 & 18.5 \\
CIRCUMBOREALES & 33.3 & 20.80 & 8.33 & 37.0 \\
SUBTROPICALES & 00 & 4.17 & 0.00 & 0.0 \\
SUBMEDITERRANEOS & 51.9 & 50.00 & 58.30 & 44.4 \\
EUMEDITERRANEOS & 0.0 & 4.17 & 0.00 & 0.0 \\
\hline
\end{tabular}

B. Espectro epiontológico.

Podemos distinguir nueve grupos representados en la Comunidad Valenciana (Tabla IX), variando la importancia de cada uno en los distintos sectores. Para el sector Valenciano-Tarraconense, la máxima representatividad la ostentan los táxones Circumboreales establecidos en la zona durante el Cuaternario con un $29.6 \%$, seguidos por los Semimediterráneos Terciarios, con un porcentaje mucho más bajo (18.5\%). En el sector Setabense, la representatividad más elevada la comparten los táxones Heterocóricos y Semimediterráneos Terciarios, con un 20.8\%, seguidos a corta distancia por los Circumboreales Cuaternarios (16.7\%). El sector Alicantino posee una amplia representación de táxones Heterocóricos Terciarios (33.3\%), siendo también importante la de táxones Mediterráneos y de Regiones Orientales Próximas (25\%). Por último, el sector Maestracense muestra un claro predominio de táxones Circumboreales Cuaternarios (33.3\%), así como de Heterocóricos y Semimediterráneos Terciarios que representan el 18.5\%. 
Tabla IX: Espectro epiontológico.

\begin{tabular}{lrrrr}
\hline \hline & VAL-T & \multicolumn{1}{c}{ SET } & ALIC & MAEST \\
\hline HETEROCORICOS T & 14.80 & 20.80 & 33.30 & 18.5 \\
CIRCUMBOREALES T. & 3.70 & 4.17 & 8.33 & 3.7 \\
SUBTROPICALES & 0.00 & 4.17 & 0.00 & 0.0 \\
MED. Y R.O.P.T. & 14.80 & 2.50 & 24.00 & 1.1 \\
LATEMEDIT. T & 11.10 & 8.33 & 8.33 & 3.7 \\
SEMIMEDIT. T. & 18.50 & 20.80 & 16.70 & 18.5 \\
EUMEDIT. T & 0.00 & 4.17 & 0.00 & 0.0 \\
CIRCUMBOREALES C. & 29.60 & 16.70 & 0.00 & 33.3 \\
LATEMEDIT. C & 7.41 & 8.33 & 8.33 & 11.1 \\
\hline
\end{tabular}

\section{Relación Terciarios/Cuatemarias.}

La relación varía claramente para cada sector reflejando, en cierto modo, los distintos avatares que han sufrido a lo largo de la historia, especialmente en lo que se refiere a la huella de las glaciaciones. Basándonos en esto, podemos distinguir dos polos claramente opuestos: por un lado el sector Alicantino, que por sus características de situación geográfica y altitud, apenas ha debido sufrir el impacto de dichas glaciaciones, como lo demuestra el hecho del alto porcentaje de táxones Terciarios que mantiene (91.7, frente al $8.33 \%$ de táxones Cuaternarios). Por otro, el sector Maestracense, que con su mayor altitud y latitud, ha visto sustituidos gran parte de sus táxones Terciarios por otros Cuaternarios tras dicho evento geológico $(55.6 \%$ de táxones Terciarios, frente a un $44.4 \%$ de Cuaternarios). Entre ambos extremos se sitúan los sectores Setabense ( $75 \%$ de Terciarios y $25 \%$ de Cuaternarios) y ValencianoTarraconense (63\% de Terciarios y 37\% de Cuaternarios), reflejando también cada uno, de acuerdo con sus características, el distinto impacto sufrido durante las glaciaciones.

\section{CONCLUSIONES}

El presente estudio nos permite llegar a la conclusión de que los sectores florísticos definidos para la Comunidad Valerciana por Costa (1986) poseen una clara identidad diferencial, apoyada por las características físico-geográficas y subrayada por la historia evolutiva de la región en los últimos períodos geológicos. Así lo demuestran, por ejemplo, el Indice de similitud con las regiones próximas, el Indice $\mathrm{m} / \mathrm{t}$, el espectro epiontológico y la relación Terciarias/Cuaternarias. Por otra parte, y en base a estos mismos datos, es posible distinguir distintos niveles de relación entre los diferentes sectores. Así, los sectores Valenciano-Tarraconense y Maestracense por un lado, y Setabense y Alicantino por otro, forman conjuntos ampliamente relacionados entre sí, como lo demuestran las tendencias secundarias continentales (Eurosiberianas) en el primero y atlánticas (Macaronésicas) en el segundo. La unión entre estos dos conjuntos se daría a nivel de los sectores Valenciano-Tarraconense y Setabense, que también comparten cierto número de características similares.

El tipo biológico predominante entre los Pteridófitos de la Comunidad Valen- 
ciana es el Hemicriptofítico, seguido a bastante distancia por el Geofítico, lo cual, si se tienen en cuenta los datos obtenidos sobre el espectro fitocenológico resulta bastante coherente con los tipos de ecosistemas ocupados, que resultan ser prioritariamente las grietas terrosas húmedas y umbrías y las fisuras de rocas verticales, seguidos a cierta distancia por los pastizales de suelos húmedos.

Desde un punto de visto fitogeográfico, los táxones predominantes son claramente Submediterráneos, lo cual resulta bastante lógico considerando la situación general del área estudiada. El segundo lugar en importancia lo ocupan los táxones Circumboreales, salvo en el sector Alicantino, donde son sustituídos por los Heterocóricos. Esto se explica fácilmente por la ausencia en dicho sector de Táxones Circumboreales Cuaternarios, que en todos los demás alcanzan una elevada representación.

Los resultados obtenidos mediante el análisis de la relación Terciarias/Cuaternarias resultan especialmente interesantes, ya que nos hacen ver la decisiva importancia que reviste la historia evolutiva de una región, y especialmente acontecimientos como las glaciaciones Cuaternarias en la composición florística de la misma. Asimismo, este tipo de análisis, pese a su sencillez, puede resultar un instrumento sumamente eficaz para detectar interrelaciones y matices diferenciales entre sectores presuntamente similares por su situación geográfica.

\section{BIBLIOGR A F I A}

COSTA, M. -1986- La Vegetación en el País Valenciano. Universitat de Valencia. Secretariado de Publicaciones.

ITO, H. -1972- Distribution of monolete and trilete ferns in easthern Asia and Northern Oceania. J. Jap. Bot. 47:321-326.

PICHI-SERMOLLI, R.E.G. -1979- A survey of the pteridological flora of the Mediterranean Region. Webbia 34:175-242.

PICHI-SERMOLLI, R.E.G., ESPAÑA, L. \& SALVO, A.E. -1988- El valor biogeográfico de la Pteridoflora Ibérica. Lazaroa 10:187-205.

SALVO, A.E. -1982- Flora Pteridofítica de Andalucía. Tesis Doctoral. Málaga.

SALVO, A.E.\& CABEZUDO, B. -1984- Bases para la utilización de los Pteridófitos en el establecimiento de Unidades Corológicas. I. Andalucía. Anales de Biología I:309-316.

SALVO, A.E.; GARCIA-VERDUGO, J.C. \& PINA, A. (en prensa): Biogeografía numérica en pteridología.

(Aceptado para su publicación el 17 de Febrero de 1989)

Dirección de los autores: J.J. HERRERO-BORGOÑON: Unidad de Estudios sobre Desertificación, CSIC, Valencia. A.M. IBARS, J. IRANZO y M. VILAR: Departamento de Biología Vegetal, Universidad de Valencia. A. PINA, J.C. GARCIA-VERDUGO y A.E. SALVO: Departamento de Biología Vegetal, Universidad de Málaga. 